\title{
L'adénosine, un anti-inflammatoire naturel
}

L'adénosine est une substance aux activités biologiques multiples; de fait, on a trouvé des récepteurs d'adénosine sur une grande variété de cellules. Les récepteurs d'adénosine ont d'abord été classés en deux types, $A_{1}$ et $\mathrm{A}_{2}$, sur la base de l'effet de l'adénosine sur l'adénylyl cyclase, l'activation des récepteurs $A_{1}$ et $A_{2}$ conduisant respectivement à une diminution et à une élévation des niveaux intracellulaires d'AMPc. La classification des récepteurs d'adénosine est aujourd'hui plus complexe depuis l'identification de sous-types des récepteurs $A_{2}\left(A_{2 a}\right.$ et $A_{2 b}$ ) et d'un récepteur $A_{3}$, et repose principalement sur la sensibilité des récepteurs à des agonistes et antagonistes spécifiques [1,2].

Le neutrophile est impliqué dans la réaction inflammatoire et il possède les récepteurs $A_{1}$ et $A_{2}$ par lesquels l'adénosine exerce des effets suppresseurs et activateurs de fonctions cellulaires. Le neutrophile se lie à l'endothélium des veinules postcapillaires irriguant le foyer inflammatoire, migre dans l'espace extravasculaire et contribue à l'élimination d'agents infectieux par la phagocytose, la dégranulation et l'explosion oxydative, mécanismes qui impliquent la libération dans le milieu de protéines et de dérivés cytotoxiques de l'oxygène (hydrogène peroxydé et anion superoxyde). Par sa liaison au récepteur $A_{1}$, l'adénosine augmente le chimiotactisme, la phagocytose et l'adhérence du neutrophile aux cellules endothéliales, alors que sa liaison au récepteur $A_{2}$ provoque une inhibition de l'explosion oxydative induite par des agonistes comme le peptide formyl-Met-Leu-Phe (ou fMLP), de même qu'une inhibition de la phagocytose et de l'adhérence.

Le mécanisme de l'effet suppresseur de l'adénosine sur le neutrophile n'a pas encore été élucidé. Il semble important de préciser ici que, bien qu'il ait été établi que l'élévation de la concentration intracellulaire d'AMPc dans le neutrophile s'accompagne que la dégranulation, la locomotion et la production de $\mathrm{H}_{2} \mathrm{O}_{2}$ (explosion oxydative), l'inhibition des fonctions cellulaires du neutrophile par l'adénosine par l'intermédiaire du récepteur $A_{2}$ ne dépend pas de la hausse de la concentration intracellulaire de l'AMPc induite par l'adénosine. Quelques études ont démontré l'inhibition par l'adénosine de l'influx de $\mathrm{Ca}^{2+}$ dans le neutrophile activé ; cet effet constitue un mécanisme potentiel de l'effet suppresseur de l'adénosine méritant d'être très sérieusement considéré, d'autant que le calcium intervient dans la modulation de nombreuses fonctions du neutrophile. Une autre hypothèse propose que l'adénosine dissocie le récepteur de l'agoniste du neutrophile (le fMLP par exemple) des éléments de transmission du signal.

Aux effets suppresseurs de l'adénosine sur le neutrophile s'ajoute l'observation importante que le neutrophile libère lui-même de l'adénosine dans le milieu extracellulaire. L'adénosine extracellulaire mesurée dans différents milieux biologiques atteint des concentrations de 0,1 à $1 \mu \mathrm{M}$, suggérant que la modulation physiologique des fonctions du neutrophile à travers le récepteur $\mathrm{A}_{2}$ (Kd $\left.0,25 \mu \mathrm{M}\right)$ est possible et même probable. A l'appui de cette assertion, l'adénosine désaminase, enzyme catalysant la transformation de l'adénosine en inosine (métabolite inactif de l'adénosine), stimule la production de l'anion superoxyde par les neutrophiles activés. De plus, l'incubation de cellules endothéliales avec des neutrophiles activés en présence d'adénosine désaminase augmente les dommages causés aux cellules endothéliales par les neutrophiles, alors que l'ajout d'un analogue de l'adénosine, la 2-chloro-adénosine, exerce un effet protecteur. Ces observations sont à l'origine de l'hypothèse d'un rôle anti-inflammatoire physiologique de l'adénosine.

Des études récentes utilisant des modèles plus complexes confortent cette hypothèse. Par exemple, lors d'une ischémie de l'intestin, l'administration topique d'adénosine réduit l'activation des neutrophiles et les dommages qui font suite à la reperfusion. L'adénosine (ou ses analogues) est également antiinflammatoire dans le modèle de l'arthrite à l'adjuvant chez le rat, ou au cours de la réaction inflammatoire induite par l'injection pleurale de carragénine. De plus, on a suggéré que le méthotrexate, un antifolate couramment utilisé comme anti-inflammatoire dans le traitement des arthropathies inflammatoires chroniques, exercerait ces effets, au moins en partie, par l'intermédiaire de l'adénosine [3]. Le méthotrexate provoque, en effet, une augmentation de la concentration d'adénosine dans les milieux inflammatoires, de même qu'une diminution de la migration des neutrophiles. Cet effet du méthotrexate sur la migration des neutrophiles in vivo pourrait être la conséquence de l'élévation de la concentration d'adénosine puisque, in vitro, des concentrations micromolaires d'adénosine inhibent l'adhérence des neutrophiles aux cellules endothéliales, étape essentielle du processus d'extravasation leucocytaire. Ainsi, ces données impliquent l'adénosine endogène dans le contrôle du processus inflammatoire, et suggèrent de nouvelles cibles pharmacologiques pour le développement d'agents anti-inflammatoires, les récepteurs de l'adénosine et/ou les enzymes impliquées dans son métabolisme [4].

P.B.

1. Collis MG, Hourani SMO. Adenosine receptor subtypes. Trends Physiol Sci 1993 ; 41: 360-6. 2. Gurden MF, Coates J, Ellis F, Evans B, Foster M Hornby E, Kennedy I, Martin DP, Strong P, Vardey $\mathrm{C}$, Wheeldon A. Functional characterization of three adenosine receptor types. BrJ Pharmacol $1993 ; 109: 693-8$

3. Cronstein BN, Naime D, Ostad E. The anti-inflammatory mechanism of methotrexate. J Clin Invest 1993 ; 92 : 2675-82.

4. Cronstein BN. Adenosine, an endogenous antiinflammatory agent. $J$ Appl Physiol $1994 ; 76: 5$-13 